\title{
Early but not late pregnancy induces lifelong reductions in the proportion of mammary progesterone sensing cells and epithelial Wnt signaling
}

\author{
Fabienne Meier-Abt ${ }^{1,2}$, Heike Brinkhaus ${ }^{1}$ and Mohamed Bentires-Alj ${ }^{1 *}$ \\ See related research by Meier-Abt et al., http://breast-cancer-research.com/content/15/2/R36
}

Early pregnancy has a strong and lifelong protective effect against breast cancer in humans and rodents [1-3]. This breast cancer protective effect is diminished or even reversed after late pregnancy [4]. As a potential mechanism for the early parity-induced protective effect, we and others have demonstrated that early parity induces differentiation of mammary epithelial cells [5-7]. Furthermore, early parity downregulates the Wnt/Notch signaling ratio and the in vitro and in vivo proliferation potential of basal stem/progenitor cells in mice [6,7]. These early parity-induced changes of gene expression and dynamics of mammary stem/progenitor cells were caused primarily by a decrease in the proportion of hormone-sensitive and Wnt4-secreting luminal epithelial cells $[6,7]$.

Although the reported findings provided a valid explanation for the breast cancer protective effect of an early pregnancy, two important questions remained unanswered. First, the early parity-induced cellular and molecular changes in mammary epithelium were investigated at a single time point (tissue/cell harvest at 40 days after weaning), leaving the question of their persistence unanswered. Second, mice were always mated at the young age of 6 weeks, and hence the effects of late pregnancy were not addressed. To directly answer these open questions, we performed additional experiments in old mice (22 months old) that had completed an early pregnancy at 6 weeks and in mice that had gone through late pregnancy (mating at

\footnotetext{
* Correspondence: Bentires@fmi.ch

${ }^{1}$ Mechanisms of Cancer, Friedrich Miescher Institute for Biomedical Research, Maulbeerstrasse 66, Basel $\mathrm{CH}-4058$, Switzerland

Full list of author information is available at the end of the article
}

24 weeks). As markers for the parity-induced decrease in mammary gland hormone responsiveness and the reduction of basal epithelial Wnt signaling [6], immunohistochemistry in mammary gland sections was performed for progesterone receptor (PR)-positive cells and for Wnt target gene expression (versican and keratin 15 [8]) as well as for epithelial cells expressing nuclear beta-catenin. Furthermore, Wnt4 expression was compared between mammary gland tissues of parous and age-matched virgin control mice.

The results demonstrate that early pregnancy-induced reductions in the proportion of PR-positive cells and expression of basal epithelial Wnt targets versican and keratin 15 persist into advanced age, and thus are of lifelong duration in mice (Figure 1A). Furthermore, pregnancy occurring at a very late age had only marginal or even no effects on PR-positive cells and expression of Wnt targets versican and keratin 15 (Figure 1B). We also noticed changes in the proportion of PR-positive cells upon aging in nulliparous mice. However, because the data in the studies on 22-month-old mice with early pregnancy and 8-month-old mice with late pregnancy were derived from two independent experiments, no direct conclusions can be drawn for the effect of age per se on the proportion of PR-positive cells in nulliparous control mice.

To further substantiate a persistent decrease of epithelial Wnt signaling by early parity, we extended the analysis to beta-catenin, a marker for active Wnt signaling. We found a persistent decrease in the proportion of nuclear beta-catenin-positive mammary epithelial cells from $11.1 \pm 1.5 \%$ in 22 -month-old age-matched virgins to $6.7 \pm 0.9 \%$ in 22 -month-old mice that had gone through pregnancy at an early age. In contrast, nuclear 
A

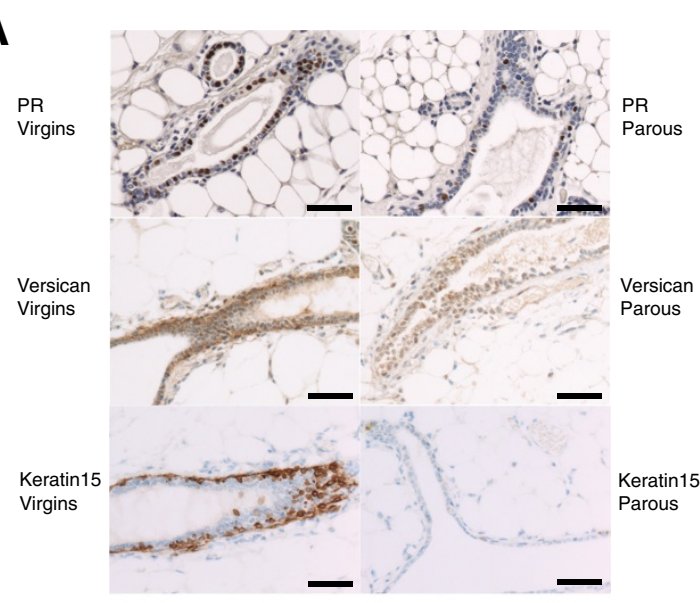

B

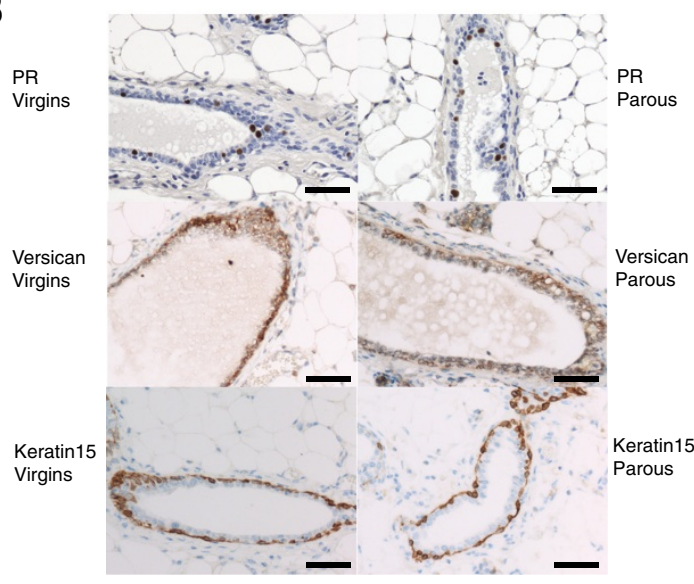

C

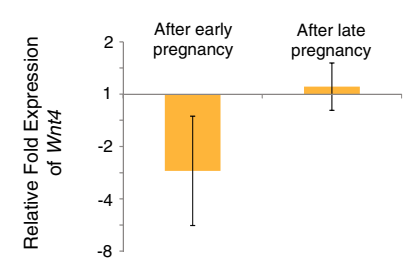

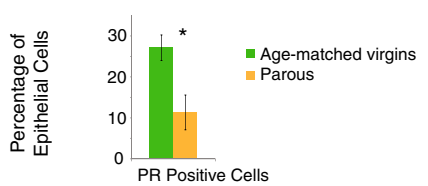

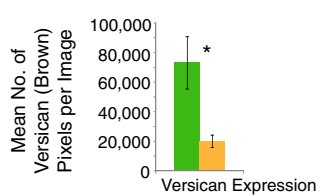
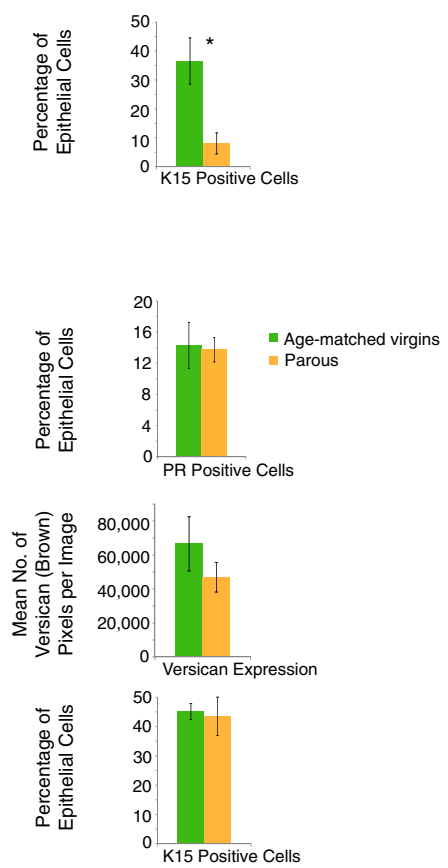

Figure 1 (See legend on next page.) 
(See figure on previous page.)

Figure 1 Effects of early and late parity on hormone (progesterone) sensitive cells and Wnt target gene (versican and keratin 15) expression in mice. (A) Early parity-induced decrease in expression of progesterone receptor (PR) and Wnt target genes versican and keratin 15 persists in old mice. Six-week-old mice (FVB/NHanHsd) were time-mated and allowed to lactate their pups for 21 days. Mammary glands were harvested after cessation of the estrus cycle when mice were 22 months old. Immunohistochemical staining of sections of paraffin-embedded mammary glands for PR, versican and keratin 15 (K15) and their quantification were performed as described previously [6]. Scale bars $=50 \mu \mathrm{m}$. For PR, quantitative data represent the mean \pm standard deviation (SD) of 2,500 counted cells from five virgin mice and five parous mice. For versican, quantitative data represent the mean \pm standard error of the mean (SEM) of 70 randomly selected images from five virgin mice and five parous mice. For keratin 15, quantitative data represent the mean \pm SD of 1,500 counted cells from three virgin mice and three parous mice. By twotailed unpaired Student $t$ test: for $P R, P=1.53 \times 10^{-4}$; for versican, $P=0.004$; for keratin $15, P=0.0049$. Primary antibodies included antiprogesterone receptor (Clone SP2, catalog number RM-9102; ThermoScientific, Lausanne, Switzerland), versican (catalog number ab1033; Millipore/Chemicon, Billerica, MA, USA), keratin 15 (EPR1614Y, catalog number ab52816; Abcam, Cambridge, UK), and secondary antibodies were biotinylated anti-rabbit lgG (catalog number BA-1000; Vector Labs, Peterborough, UK) and anti-rabbit horseradish peroxidase-conjugated polymer Immpress (catalog number MP-7401; Vector Labs). (B) Late pregnancy does not significantly decrease the proportion of PR-positive cells and the expression of Wht targets versican and keratin 15. Twenty-four-week-old mice were time-mated and allowed to lactate their pups for 21 days. The postweaning period until gland harvest was identical to the early parity protocol (that is, 40 days) as described previously [6]. Immunohistochemical staining was performed as in (A). Scale bars $=50 \mu \mathrm{m}$. For PR, quantitative data represent the mean \pm SD of 2,500 counted cells from five virgin mice and five parous mice. For versican, quantitative data represent the mean \pm SEM of 70 randomly selected images from five virgin mice and five parous mice. For keratin 15, quantitative data represent the mean \pm SD of 1,500 counted cells from three virgin mice and three parous mice. By two-tailed unpaired Student $t$ test: for $P R, P=0.72$; for versican, $P=0.28$; for keratin $15, P=0.71$. (C) Early pregnancy leads to a persistent decrease in Wnt4 expression in snap-frozen mammary glands. In contrast, no change in Wnt4 expression was observed after late pregnancy. Quantitative polymerase chain reaction was performed on snap-frozen mammary glands from parous and age-matched virgin control mice, using Hprt as a reference gene. Data are presented as fold expression in mammary glands from parous mice relative to age-matched virgin control mice. Data represent the mean \pm SD of tissues from seven to 10 mice per group.

beta-catenin in epithelial cells was not affected by late pregnancy $(10.8 \pm 1.3 \%$ in age-matched virgins versus $9.6 \pm 1.9 \%$ in parous mice) (data represent the mean \pm standard deviation of 3,000 counted cells from six mice per group). Furthermore, we had previously shown that decreases in PR-positive cells were associated with decreases in Wnt4 expression, which in turn was associated with decreased Wnt signaling in basal stem/ progenitor cells [6]. Consistent with the data for PR, the basal epithelial Wnt targets versican and keratin 15; and for nuclear beta-catenin in epithelial cells, Wnt4 expression was also persistently reduced after early pregnancy but remained unchanged after late pregnancy (Figure 1C).

Together with the previously reported study [6], the findings presented here represent the first direct comparison of the effects of early and late pregnancy on hormone sensing cells and on epithelial Wnt signaling in the mammary gland. The data conform with the presence of parity-induced changes in the dynamics of mammary cell functions upon early pregnancy [9] and suggest their absence upon late pregnancy in mice $[10,11]$. Overall these results are fully consistent with the lifelong breast cancer protective effect of early but not late pregnancy in humans [7]. They reinforce the validity of the adopted mouse model to study prevention strategies against human breast cancer and highlight the need for further investigations into the molecular changes following late versus early pregnancy.

\section{Abbreviations}

PR: Progesterone receptor.

\section{Competing interests}

The authors declare that they have no competing interests.

\section{Author details}

${ }^{1}$ Mechanisms of Cancer, Friedrich Miescher Institute for Biomedical Research, Maulbeerstrasse 66, Basel CH-4058, Switzerland. 'Faculty of Science,

University of Basel, Klingelbergstrasse 50, Basel CH-4056, Switzerland.

\section{Published: 17 Mar 2014}

\section{References}

1. MacMahon B, Cole P, Lin TM, Lowe CR, Mirra AP, Ravnihar B, Salber EJ, Valaoras VG, Yuasa S: Age at first birth and breast cancer risk. Bull World Health Org 1970, 43:209-221.

2. Albrektsen G, Heuch I, Hansen S, Kvale G: Breast cancer risk by age at birth, time since birth and time intervals between births: exploring interaction effects. Br J Cancer 2005, 92:167-175.

3. Medina D: Mammary developmental fate and breast cancer risk. Endocr Relat Cancer 2005, 12:483-495.

4. Britt K, Ashworth A, Smalley M: Pregnancy and the risk of breast cancer. Endocr Relat Cancer 2007, 14:907-933.

5. Russo $I H$, Russo J: Pregnancy-induced changes in breast cancer risk. J Mammary Gland Biol Neoplasia 2011, 16:221-233.

6. Meier-Abt F, Milano E, Roloff T, Brinkhaus H, Duss S, Meyer DS, Klebby I, Balwierz PJ, van Nimwegen E, Bentires-Alj M: Parity induces differentiation and reduces Wnt/Notch signaling ratio and proliferation potential of basal stem/progenitor cells isolated from mouse mammary epithelium. Breast Cancer Res 2013, 15:R36.

7. Meier-Abt F, Bentires-Alj M: How pregnancy at early age protects against breast cancer. Trends Mol Med 2013, 13:143-153.

8. Palmer HG, Anjos-Afonso F, Carmeliet G, Takeda H, Watt FM: The vitamin D receptor is a Wnt effector that controls hair follicle differentiation and specifies tumor type in adult epidermis. PloS One 2008, 3:e1483.

9. Siwko SK, Dong J, Lewis MT, Liu H, Hilsenbeck SG, Li Y: Evidence that an early pregnancy causes a persistent decrease in the number of 
functional mammary epithelial stem cells: implications for pregnancyinduced protection against breast cancer. Stem Cells 2008,

26:3205-3209

10. Britt KL, Kendrick H, Regan JL, Molyneux G, Magnay FA, Ashworth A, Smalley MJ: Pregnancy in the mature adult mouse does not alter the proportion of mammary epithelial stem/progenitor cells. Breast Cancer Res 2009, 11:R20.

11. Raafat A, Strizzi L, Lashin K, Ginsburg E, McCurdy D, Salomon D, Smith GH, Medina $\mathrm{D}$, Callahan R: Effects of age and parity on mammary gland lesions and progenitor cells in the FVB/N-RC mice. PLoS One 2012. 7:e43624.

\section{$10.1186 /$ bcr3626}

Cite this article as: Meier-Abt et al: Early but not late pregnancy induces lifelong reductions in the proportion of mammary progesterone sensing cells and epithelial Wnt signaling. Breast Cancer Research 2014, 16:402 